\title{
New records of Hydraenidae and Elmidae (Coleoptera) from Russia and adjacent countries
}

\author{
Alexander A. ProKIN ${ }^{1}$, Stanislav V. LITOVKIN ${ }^{2}$ and Manfred A. JÄCH ${ }^{3}$ \\ ${ }^{1}$ Papanin Institute for Biology of Inland Waters, Russian Academy of Sciences, Borok, Nekouzsky District, 152742 \\ YaroslavlOblast',Russia; prokina@mail.ru \\ ${ }^{2}$ Russian Entomological Society, Samara,Russia; sats.lit@gmail.com \\ ${ }^{3}$ Naturhistorisches Museum Wien, Burgring 7, A-1010 Wien, Austria; manfred.jaech@nhm-wien.ac.at
}

\begin{abstract}
New data on the distribution of 27 species of Hydraenidae and three species of Elmidae (Coleoptera) from Russia, Ukraine, Kazakhstan and Uzbekistan are provided. Ochthebius foveolatus Germar, 1824 and Stenelmis koreana Satô, 1978 are recorded from Russia for the first time. Other new records are: Ochthebius bernhardi Jäch et Delgado, 2008 (Ukraine), O. depressus Sahlberg, 1900 (Ural Mountains), O. erzerumi Kuwert, 1887 (European part of Russia and Ural Mountains), O. flavipes Dalla Torre, 1877 (Ural Mountains), O. jermakovi Orchymont, 1933 (Kazakhstan), and O. joosti Jäch, 1992 (Kazakhstan and Uzbekistan). A new record for the rarely collected Laeliaena sparsa Sahlberg, 1900 from Uzbekistan is also provided. For Ochthebius yoshitomii Jäch et Delgado, 2014 new records and information on the correct type locality, as well as detailed habitat information and photographs of its habitat on Kunashir Island are provided.
\end{abstract}

Key words: beetles, new records, Russia, Ukraine, Kazakhstan, Uzbekistan, Ural, Kunashir Island

\section{INTRODUCTION}

The distribution of the Hydraenidae and Elmidae of Russia and other countries of the former Soviet Union is still poorly known. Many species are known only from the type locality or from very few localities. In this paper we present several new and other interesting records, trying to fill some gaps.

In the Catalogue of Palaearctic Coleoptera (Jäch, Skale 2015) several species of Hydraenidae were recorded from some of the countries of the former Soviet Union for the first time. Here we provide detailed locality data, on which these new records were based, using the same abbreviations for subdivisions of Russia as in the Catalogue, except for the Ural Mountains (Chelyabinsk Oblast'), because they form a natural border between Europe and Asia and are thus treated as a separate subdivision herein.

The information presented here will be useful to clarify the ranges of species, and it will provide the base for their protection at a regional level.

\section{MATERIAL}

This paper is based mainly on material collected by two of the authors, and on material from the Zoological Museum, Lomonosov Moscow State University (Moscow, Russia), Naturhistorisches Museum Wien (Austria), Moscow State Pedagogical University (Moscow, Russia) and several private collections.

The depository of the specimens examined is abbreviated using the following acronyms:

APC private collection of Alexander Prokin (Voronezh, Russia)

DPC private collection of Dmitry Palatov (Moscow, Russia)

ISC private collection of Igor Solodovnikov (Vitebsk, Belarus)

MPU Moscow State Pedagogical University (Moscow, Russia) 
NMW Naturhistorisches Museum Wien (Vienna, Austria)

SLC private collection of Stanislav Litovkin (Samara, Russia)

YKC private collection of Yakov Kovalenko (Moscow, Russia)

ZMUM Zoological Museum, Lomonosov Moscow State University (Moscow, Russia)

\section{RESULTS}

Family Hydraenidae Mulsant, 1844

\section{Aulacochthebius narentinus (Reitter, 1885)}

(Figs 1-2)

Recorded from Orenburg Oblast' (ST) for the first time by Litovkin (2012b).

Russia (CT): 52 55'58.8"N, 49³8'55.5"E, Samara Oblast', Bezenchuk District, near Chapaevsk, small lake in Chapaevka River flood-plane, 2-4 Jul 2013, leg. S. Litovkin, 10 ex. (7 ex. - SLC, 2 ex. - NMW, 1 ex. - ISC); same locality data, 10 Aug 2015, leg. S. Litovkin, 3 ex. (SLC).

This is the second record from Russia, and the first record from the central part of European Russia (CT).

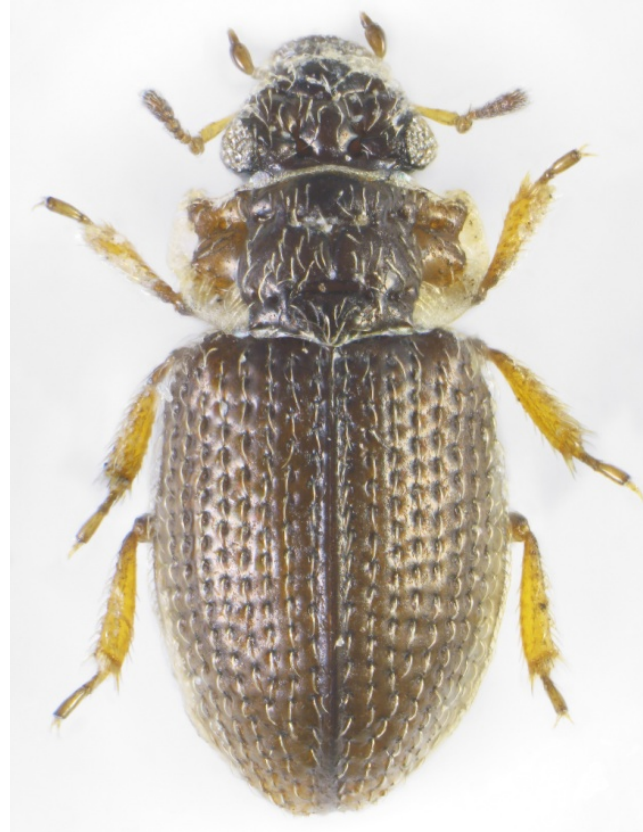

Fig. 1. General habitus of Aulacochthebius narentinus (Reitter, 1885). Photo by S. Litovkin.

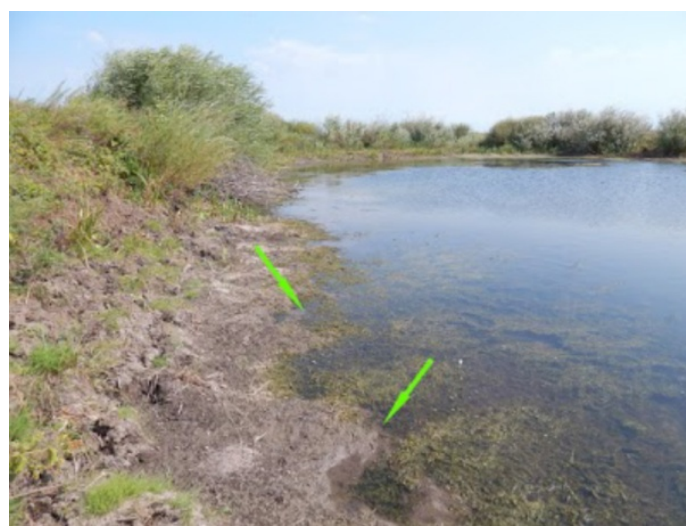

Fig. 2. Habitat of A. narentinus in Samara Oblast' Photo by S. Litovkin. Arrows indicate actual choriotope.

\section{Hydraena (s.str.) canakcioglui aydini Janssens, 196}

Russia (ST): Krasnodarsky Kray, Agura River, $50 \mathrm{~m}$ upstream of waterfalls, on stones with moss under water, 7 Mar 2006, leg. P. Petrov, 2 ex. (APC); the record for ST in Jäch, Skale (2015) is based on these specimens. 


\section{Hydraena (s.str.) gracilis Germar, 1824}

Recently recorded from ST by Litovkin (2012a).

Russia (ST): Orenburg Oblast', Orenburg Nature Reserve, NE of Kuskol' Lakes, 3 May 2011, leg. P. Petrov, 1 ex. (APC).

ST should be added to the Catalogue of Palaearctic Coleoptera.

\section{Hydraena (s.str.) pulchella Germar, 1824}

Russia (ST): Belgorod Oblast', Olshanka River, near bridge, 7 Jun 2011, leg. A. Silina, 1 ex. (APC); (CT): Yaroslavl Oblast', Nekouzsky District, near Borok, Latka River, 15 Aug 2004, leg. N. Zhgareva, 1 ex., Ild' River, 29 Jul 2010, leg. N. Zhgareva, 1 ex., 29 Aug 2010, leg. N. Zhgareva, 1 ex. (APC) (Prokin et al., 2013); Samara Oblast', Elkhovka District, near Proleyka, stream, 5355'59"N, 50¹4'30"E, 25 Jul 2005, leg. S. Litovkin, 1 ex. (SLC).

ST should be added to the Catalogue of Palaearctic Coleoptera.

\section{Laeliaena sparsa Sahlberg, 1900}

Uzbekistan: $40^{\circ} 34^{\prime} 50.9^{\prime \prime} \mathrm{N}, 6^{\circ} 39^{\prime} 26.2^{\prime \prime} \mathrm{E}$, Navoiy Province, Nurata District, Nuratau Mts., near Sentob, 16 Apr 2014, leg. Ya. Kovalenko, in mountain river on stones, 4 ex. (2 ex. YKC, 2 ex. - APC).

\section{Micragasma paradoxum Sahlberg, 1900}

(Figs 3-4)

Russia (ST): 4907'43.6"N, 46²4'39.5"E, Volgograd Oblast', $18 \mathrm{~m}$ below sea level, near Lake Elton, residual pools in temporary watercourse, 17 Apr 2008, leg. A. Prokin, 7 ex. (NMW).

Makarov et al. (2009) recorded this species from the vicinity of Lake Elton under the name Micragasma cf. paradoxum Sahlberg, 1900.

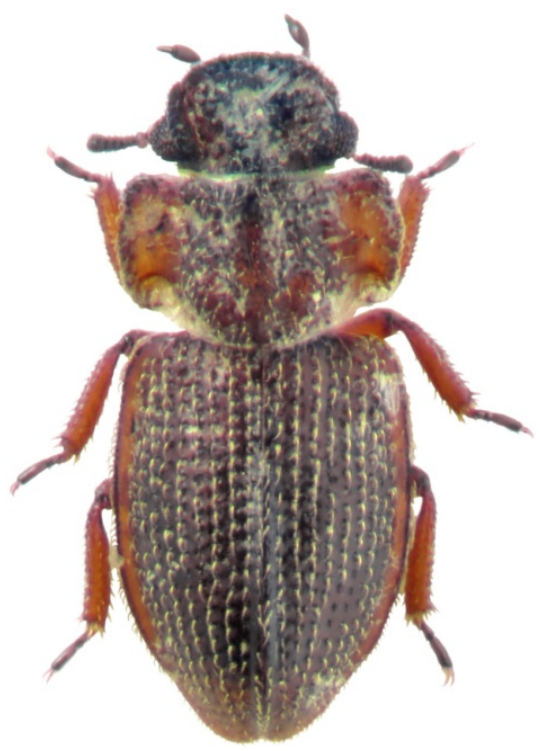

Fig. 3. General habitus of Micragasma paradoxum Sahlberg, 1900. Photo by K. Makarov.

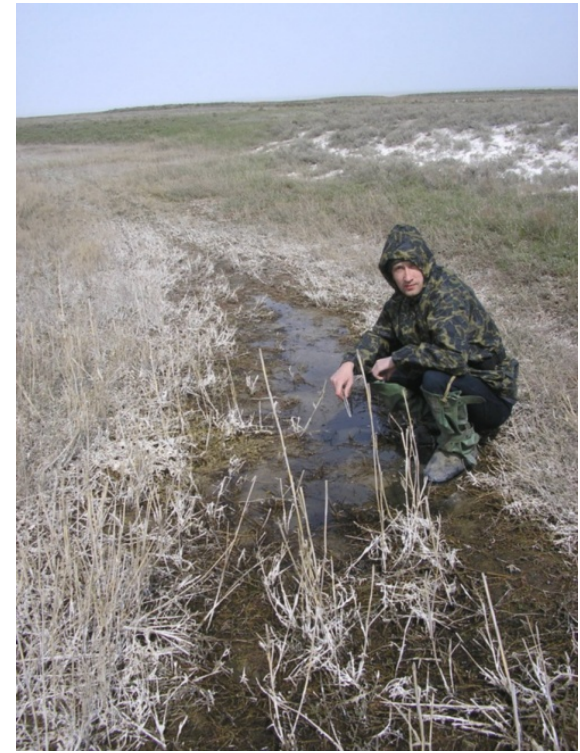

Fig. 4. Habitat of M. paradoxum in Volgograd Oblast', where A. Prokin collected specimens in 2008. Photo by A. Kutuzov. 


\section{Ochthebius (Asiobates) depressus Sahlberg, 1900}

Russia (Ural): Chelyabinsk Oblast', Ural River at left bank, 5 km S Cheka Hill, 15-16 Jul 2007, leg. P. Petrov, 1 ex. (NMW); Kazakhstan: 43²1'52.5"N, 7003'49.0"E, 930 m, Karatau Ridge, 13 km NE Bayzhansay, stream, in gravel, 29-30 May 2015, leg. S. Litovkin, 15 ex. (SLC); 4251'49.7"N, 6951'58.7"E, 540 m, Boraldaytau Mts., 7.4 km ENE Terekty, backwater of Boralday River, 10 Jun 2015, leg. S. Litovkin, 13 ex. (SLC).

This is the first record for the Ural Mountains.

\section{Ochthebius (Asiobates) flavipes Dalla Torre, 1877}

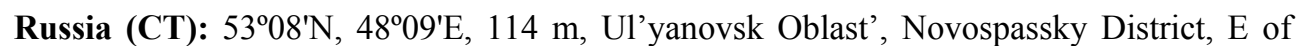
Maryevka Village, 07 Jul 2009, leg. A.V. Kovalev, 3 ex. (APC); 5313'24"N, 50³2'42"E, Samara Oblast', Volzhskiy District, $3.5 \mathrm{~km}$ SE Alekseevka, waterbody in Samara River floodplane, 28 Apr 2012 and 11 May 2012, leg. S. Litovkin, 2 ex. (SLC); Ural: Chelyabinsk Oblast', near Cheka Mountain, 06 May 2006, leg. I. Fedorov, 1 ex. (APC).

New for the Ural Mountains.

\section{Ochthebius (Asiobates) nonaginta Jäch, 1998}

(Figs 5-6)

Russia (ST): 50³7'38.8"N, 54²2'55.4"E, 190 m, Orenburg Oblast', Sol'-Iletsk District, $11 \mathrm{~km}$ SW Troitsk, steppe, hills, in slow stream, 18-21 May 2012, leg. S. Litovkin, 1 ex. (SLC); 51 ${ }^{\circ} 22^{\prime} 36^{\prime \prime} \mathrm{N}, 55^{\circ} 36^{\prime} 12^{\prime \prime} \mathrm{E}, 180 \mathrm{~m}$, Akbulak District, $3 \mathrm{~km} \mathrm{~N}$ Akbulak, steppe, pool, 20 21 Apr 2013, leg. S. Litovkin, 1 ex. (SLC).

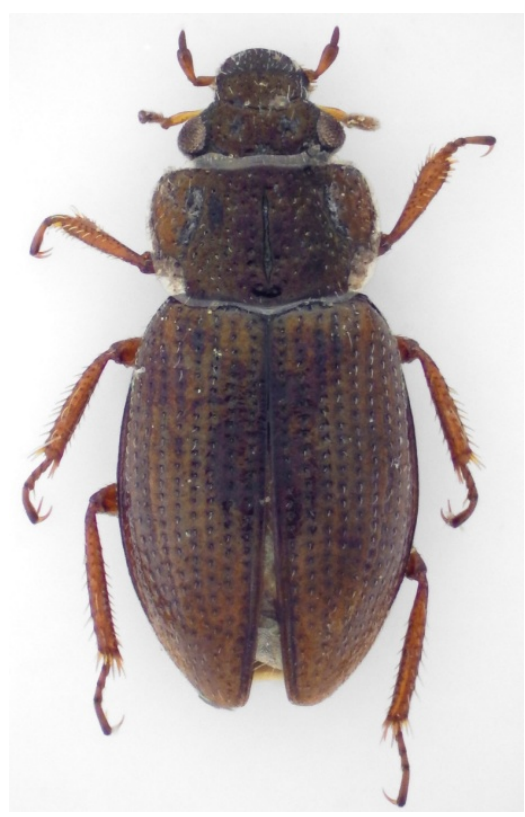

Fig. 5. General habitus of Ochthebius nonaginta Jäch, 1998. Photo by S. Litovkin.

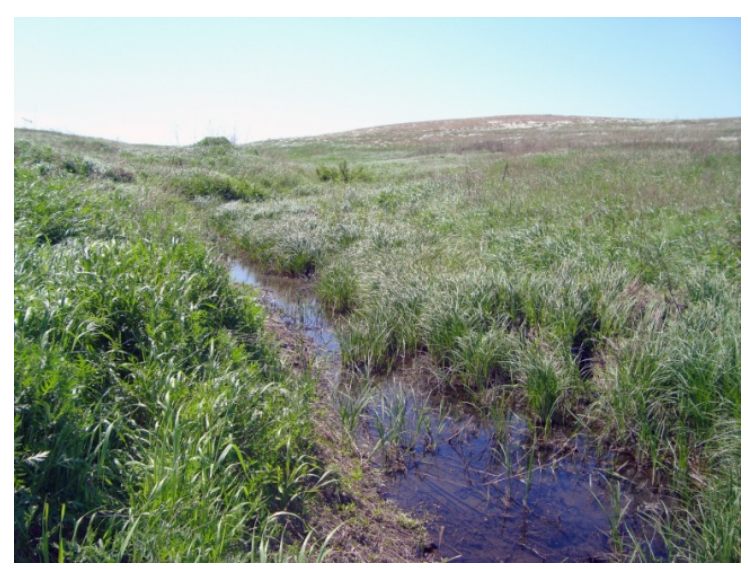

Fig. 6. Habitat of of $O$. nonaginta in Orenburg Oblast' $(11 \mathrm{~km}$ SW Troitsk). Photo by S. Litovkin. 


\section{Ochthebius (Asiobates) minimus (Fabricius, 1792)}

Recorded from Kazakhstan for the first time by Temreshev (2012).

Kazakhstan: $50^{\circ} 19^{\prime} 03.8^{\prime \prime} \mathrm{N}, 57^{\circ} 26^{\prime} 09.6^{\prime \prime} \mathrm{E}, 224 \mathrm{~m}$, Aktyubinsk Oblast', E of Aktobe (= Aktyubinsk), Zhaman-Karagaly River, 30 Apr 2015, leg. S. Litovkin, 3 ex. (SLC).

KZ (= Kazakhstan) should be added to the Catalogue of Palaearctic Coleoptera.

\section{Ochthebius (Asiobates) perdurus Reitter, 1899}

Kazakhstan: $43^{\circ} 46^{\prime} 25.4^{\prime \prime} \mathrm{N}, 68^{\circ} 48^{\prime} 44.6^{\prime \prime} \mathrm{E}, \sim 950$ m,Karatau Ridge, Kelinshektau Mountain, 7.3 km SW Abay, mountain stream, 15-16 May 2015, leg. S. Litovkin, 1 ex. (SLC).

In Kazakhstan this species was previously known only from the surroundings of Almaty (“Alma Ata”) and Karkaraly (“Karkaralinsk”) (Jäch, 1990).

\section{Ochthebius (Asiobates) remotus Reitter, 1885}

Recorded from the southern part of European Russia (ST) by Litovkin (2012a).

Russia (ST): 5057'06.1"N, 54²16'22.1"E, 80 m, Orenburg Oblast', Sol'-Iletsk District, 5.5 km SE Novoiletsk, Ilek River, 22-24 May 2012, leg. S. Litovkin, 3 ex. (SLC); (CT): Samara Oblast', Kinel' District, 10 km SE Malaya Malyshevka, bank of Samara River, 15 Aug 2005, leg. S. Litovkin, 3 ex. (SLC); 5317'10.8"N, 5056'45.6"E, Samara Oblast', Kinel' District, vicinity of Vertyaevka, Bol'shoy Kinel' River, 10 Jun 2013, leg. S. Litovkin, 5 ex. (SLC); $53^{\circ} 13^{\prime} 50.2^{\prime \prime N}, 51^{\circ} 10^{\prime} 42.2^{\prime \prime E}, 50 \mathrm{~m}$, Samara Oblast', Kinel'-Cherkassy District, $5 \mathrm{~km} \mathrm{~S}$ Krotovka, Kutuluk River, 23 Jul 2013, leg. S. Litovkin, 10 ex. (8 ex. - SLC, 2 ex. - ISC); $53^{\circ} 08^{\prime} 41.5^{\prime \prime N}, 48^{\circ} 10^{\prime} 54.3^{\prime \prime E}$, Samara Oblast' near border of Ul'yanovsk Oblast', Syzran' District, 2 km SSE Kuropatkino, Syzranka River, sandbank, 12 Jul 2015, leg. S. Litovkin, 3 ex. (SLC); 53¹7'03.6"N, 5050'30.2"E, 15 Aug 2015, Samara Oblast', Kinel' District, near Pokrovka, Bol'shoy Kinel' River, leg. S. Litovkin, 5 ex.

ST should be added to the Catalogue of Palaearctic Coleoptera.

\section{Ochthebius (s.str.) bellstedti Jäch, 1992}

Russia (ST): $50^{\circ} 37^{\prime} 38.8^{\prime \prime} \mathrm{N}, 5^{\circ} 28^{\prime} 55.4^{\prime \prime E}, 190 \mathrm{~m}$, Orenburg Oblast', Sol'-Iletsk District, $11 \mathrm{~km}$ SW Troitsk, steppe, hills, in slow stream, 18-21 May 2012, leg. S. Litovkin, 1 ex. (SLC). Kazakhstan: 46 $58^{\prime} 11.0^{\prime \prime} \mathrm{N}, 61^{\circ} 29^{\prime} 27.5^{\prime \prime} \mathrm{E}, 80 \mathrm{~m}$, Kyzylorda Oblast', $21 \mathrm{~km}$ NW Aral (= Aral'sk), desert, in brackish pools, 1 May 2015, leg. S. Litovkin, 9 ex. (SLC); 435' $58.1^{\prime \prime} \mathrm{N}$, $67^{\circ} 29^{\prime} 04.9^{\prime E}$, $240 \mathrm{~m}$, Karatau Ridge foothill, $3.5 \mathrm{~km}$ NE Birlik, stream, 29 Apr 2013, leg. S.

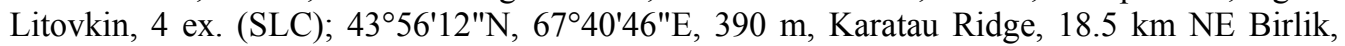
Akuyik River valley, 29 Apr-2 May 2013, leg. S. Litovkin, 6 ex. (SLC); 433' $26^{\prime \prime} \mathrm{N}$, $68^{\circ} 31$ '56"E, $\sim 600 \mathrm{~m}$, Karatau Ridge, 8-10 km N Kentau, Bayaldyr River valley, 25 May 2013, leg. S. Litovkin, 4 ex. (SLC); 4336'48"N, 6902'38"E, 710 m, Karatau Ridge, 19 km SW Sholakkorgan, shallow stream, 14 May 2015, leg. S. Litovkin, 1 ex. (SLC); 42॰51'47"N, 6952'01"E, 550 m, Boraldaytau Mts., 7.4 km ENE Terekty, Boralday River valley, 23-26 May 2015, leg. S. Litovkin, 1 ex. (SLC); 43²1'52.5"N, 7003'49.0"E, $930 \mathrm{~m}$, Karatau Ridge, 13 km NE Bayzhansay, stream, in gravel, 29-30 May 2015, leg. S. Litovkin, several specimens (SLC); 4329'43"N, 70³9'37"E, $375 \mathrm{~m}$, Zhambyl Oblast', vicinity of Ashchykol' (salt lake), brackish pools, 3 Jun 2015, leg. S. Litovkin, 20 ex. (SLC);

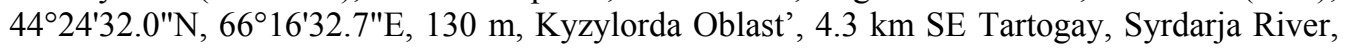
13 Jun 2015, leg. S. Litovkin, 1 ex. (SLC). 


\section{Ochthebius (s.str.) bernhardi Jäch et Delgado, 2008}

Ukraine: Nikolaevsk Oblast', Kinburn Spit, saline lake, 29 Jun 2007, leg. V. Dyadichko, 1 ex. (APC), 1 May 2007, reed bog in steppe, 1 ex. (APC); Russia (ST): $51^{\circ} 09^{\prime} 09.5^{\prime \prime} \mathrm{N}$, $55^{\circ} 00^{\prime} 22.6^{\prime \prime E}, 114 \mathrm{~m}$, Orenburg Oblast', Sol'-Iletsk District, Sol'-Iletsk, Peschanka River, near hypersaline lakes, 18 May 2012, leg. S. Litovkin, 4 ex. (SLC); 5057'06.1"N, 54¹6'22.1"E, 80 m, Orenburg Oblast', Sol'-Iletsk District, 5.5 km SE Novoiletsk, Ilek River, 22-24 May 2012, leg. S. Litovkin, 1 ex. (SLC); 5057'25.0"N, 54²15'50.3"E, 85 m, Orenburg Oblast', Sol'-Iletsk District, $4.8 \mathrm{~km}$ SE Novoiletsk, oxbow in Ilek River flood-plane, 24 May 2012, leg. S. Litovkin, 1 ex. (SLC); 5055'48.9"N, 55 53'25.5"E, Orenburg Oblast', Akbulak District, 18.5 km SE Akbulak, near Karabutak River, at light, 15 Jun 2015, leg. S. Litovkin, 3 ex. (SLC); (CT): $52^{\circ} 55^{\prime} 58.8^{\prime \prime} \mathrm{N}, 49^{\circ} 38^{\prime} 55.5^{\prime \prime E}$, Samara Oblast', Bezenchuk District, near Chapaevsk, small lake in Chapaevka River flood-plane, 2-4 Jul 2013, leg. S. Litovkin, 3 ex. (SLC); same locality data, 10 Aug 2015, leg. S. Litovkin, 3 ex. (SLC).

Records of $O$. viridis and $O$. viridis species-complex from Russia (ST): Astrakhan, Volgograd, Voronezh and Belgorod Oblast' (Jäch, 1992a; Jäch, Prokin, 2005; Silina, Prokin, 2011) refer to this species.

New for Ukraine!

\section{Ochthebius (s.str.) depressionis Jäch, 1991}

This species was known only from the type locality near Lake Elton in Volgograd Oblast' (ST) (Jäch, 1991), from recent records from Orenburg Oblast' (ST), and from eastern Kazakhstan (Zinchenko, 2015).

Russia (ST): Volgograd Oblast', near Lake Elton, pool in Lantsug River flood-plane, 14 Apr 2008, leg. A. Prokin, 6 ex. (APC); 51 $09^{\prime} 09.5^{\prime \prime N}, 55^{\circ} 00^{\prime} 22.6 " \mathrm{E}, 114 \mathrm{~m}$, Orenburg Oblast', Sol'-Iletsk District, Sol'-Iletsk, Peschanka River, near hypersaline lakes, 18 May 2012, leg. S. Litovkin, 6 ex. (4 ex. - SLC, 2 ex. - NMW).

\section{Ochthebius (s.str.) erzerumi Kuwert, 1887}

Russia (ST): Volgograd Oblast', near Lake Elton, pool in Lantsug River flood-plane, 14 Apr 2008, leg. A. Prokin, 1 ex. (APC); 50³7'38.8"N, 54²8'55.4"E, 190 m, Orenburg Oblast', Sol'-Iletsk District, 11 km SW Troitsk, steppe, hills, in slow stream, 18-21 May 2012, leg. S. Litovkin, 2 ex. (SLC); 5059'30.2"N, 54¹4'28.7"E, 80 m, Orenburg Oblast', Sol'-Iletsk District, $1 \mathrm{~km}$ E Novoiletsk, steppe, pools, 23 May 2012, leg. S. Litovkin, 20 ex. (SLC); 50 55'48.9"N, 5553'25.5"E, Orenburg Oblast', Akbulak District, 18.5 km SE Akbulak, near Karabutak River, at light, 15 Jun 2015, leg. S. Litovkin, 7 ex. (SLC); Ural: Chelyabinsk Oblast', Kizil'skoe District, near Aleksandrovsky, Karaganka River, 16 Aug 1998, leg. P. Petrov, 3 ex. (APC), Kizil'skoe District, 10 km S Cheka Hill, Ural River, 03 May 2006, leg. P. Petrov, 1 ex. (APC).

New for European Russia and Ural Mountains!

\section{Ochthebius (s.str.) evanescens Sahlberg, 1875}

Recorded from the eastern part of Kazakhstan for the first time by Zinchenko (2015).

Russia (ST): Volgograd Oblast', Lake Elton, pools near Lantsug River, 14 Apr 2008, leg.

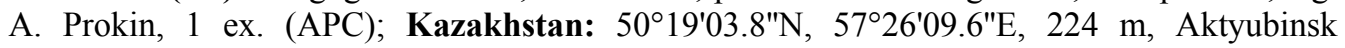
Oblast', E of Aktobe (= Aktyubinsk), Zhaman-Karagaly River, 30 Apr 2015, leg. S. Litovkin, 1 ex. (SLC); 46 $58^{\prime} 11.0^{\prime \prime} \mathrm{N}, 61^{\circ} 29^{\prime} 27.5^{\prime \prime E}, 80 \mathrm{~m}$, Kyzylorda Oblast', 21 km NW Aral (= Aral'sk), desert, in brackish pools, 1-2 May 2015, leg. S. Litovkin, 4 ex. (SLC). 


\section{Ochthebius (s.str.) fausti Sharp, 1887}

In Russia this species was known only from the type locality in Orenburg Oblast' (ST) (Jäch 1991).

Russia (ST): $50^{\circ} 57^{\prime} 06.1^{\prime \prime N}$, 54¹6'22.1"E, 80 m, Orenburg Oblast', Sol'-Iletsk District, 5.5 km SE Novoiletsk, Ilek River, 22-24 May 2012, leg. S. Litovkin, 2 ex. (SLC).

\section{Ochthebius (s.str.) foveolatus Germar, 1824}

(Figs 7-8)

Russia (CT): $53^{\circ} 08^{\prime} 41.5^{\prime \prime N}, 48^{\circ} 10^{\prime} 54.3^{\prime \prime E}$, Samara Oblast' near border of Ul'yanovsk Oblast', Syzran' District, 2 km SSE Kuropatkino, Syzranka River, sandbank, 12 Jul 2015, leg. S. Litovkin, 25 ex. (SLC).

New for Russia!

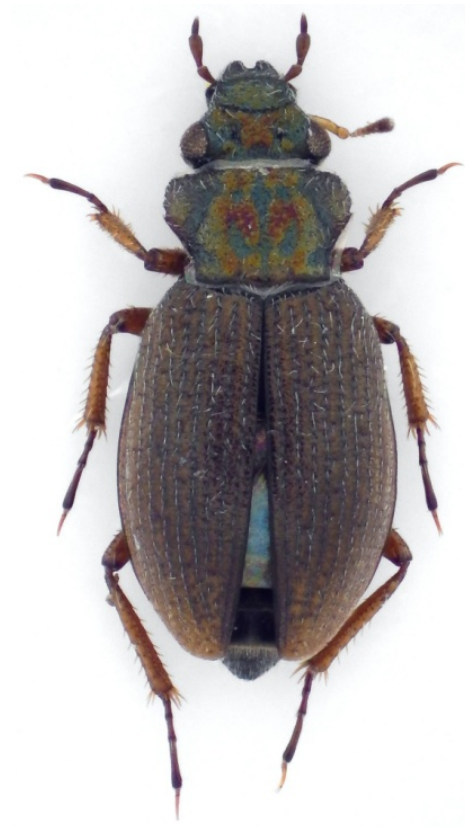

Fig. 7. General habitus of Ochthebius foveolatus Germar, 1824. Photo by S. Litovkin.

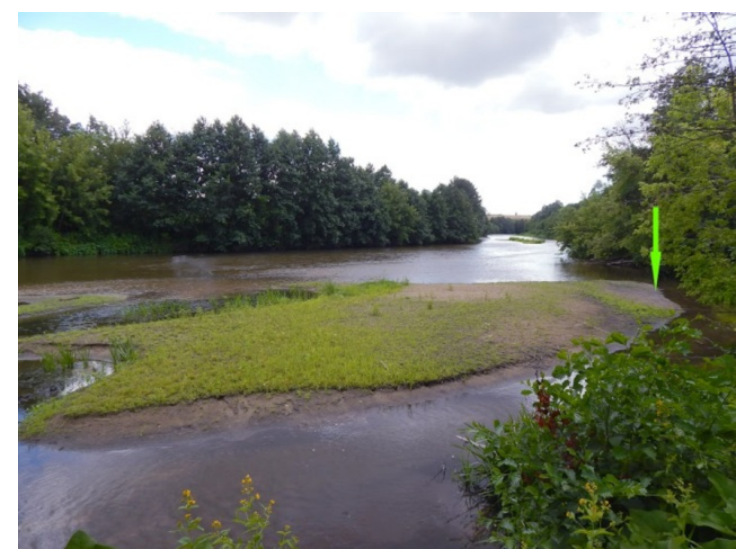

Fig. 8. Habitat of O. foveolatus in Samara Oblast'. Arrow indicates actual collecting site. Photo by S. Litovkin).

\section{Ochthebius (s.str.) hauseri Jäch, 1992}

Recorded from southern Kazakhstan ["Dzhambul, Syr Darja”] (Jäch, 1992b).

Kazakhstan: $43^{\circ} 37^{\prime} 26^{\prime \prime} \mathrm{N}, 68^{\circ} 31^{\prime} 56^{\prime \prime E}, \sim 600 \mathrm{~m}$, Karatau Ridge, 8-10 km N Kentau, Bayaldyr River valley, 2-5 May 2013, leg. S. Litovkin, 2 ex. (SLC).

\section{Ochthebius (s.str.) jermakovi d'Orchymont, 1933}

Kazakhstan: $46^{\circ} 09^{\prime} \mathrm{N}, 5^{\circ} 23^{\prime} \mathrm{E}$, Caspian Sea, Tengiz-Prorva gas deposit, 06 Nov 2014, leg. D. Smirnova, 10 ex. (8 ex. - APC, 2 ex. - NMW)

New for Kazakhstan! 


\section{Ochthebius (s.str.) joosti Jäch, 1992}

Russia (ST): 4907'43.6"N, 4647'39.5"E, Volgograd Oblast', Lake Elton, pools, 17.04.2008, leg. A. Prokin, 3 ex. (APC), pools near Lantsug River, 14 Apr 2008, leg. A. Prokin, 10 ex. (APC), 4907'43.6"N 46² $7^{\circ} 39.5^{\prime \prime} \mathrm{E},-18 \mathrm{~m}$, near Lake Elton, residual pools of temporary water course, 17 Apr 2008, leg. A. Prokin, 10 ex. (APC); Astrakhan Oblast', 7 km NW Bolshoye Bogdo Rock (mountain), 4-5 May 2006, leg. A.V. Kovalev, 2 ex. (APC); the record for ST in Jäch, Skale (2015) is based on these specimens; Uzbekistan: 38 $57^{\prime} 21.0^{\prime \prime} \mathrm{N}$, 64²'19.6"E, Qashqadaryo Province, Mirishkor District, near Urtabulak mine, 14 Apr 2014, leg. Ya. Kovalenko, 1 ex. (YKC); Kazakhstan: 4329'43"N, 70³9'37"E, 375 m, Zhambyl Oblast', vicinity of Ashchykol' (salt lake), brackish pools, 3 Jun 2015, leg. S. Litovkin, 38 ex. (30 ex. - SLC, 8 ex. - NMW).

New for Uzbekistan and Kazakhstan!

\section{Ochthebius (s.str.) meridionalis Rey, 1885}

Recorded from Kazakhstan for the first time by Zinchenko (2015).

Russia (ST): Volgograd Oblast', near Lake Elton, pool in Lantsug River flood-plane, 14 Apr 2008, leg. A. Prokin, 1 ex. (APC); 5055'48.9"N, 55 53'25.5"E, Orenburg Oblast', Akbulak District, 18.5 km SE Akbulak, near Karabutak River, at light, 15 Jun 2015, leg. S. Litovkin, 1 ex. (SLC); Kazakhstan: 4324'50.5"N, 70³8'12.6"E, 400 m, Zhambyl Oblast', W bank of Akkol' Lake, 18 May 2015, leg. S. Litovkin, 20 ex. (SLC).

\section{Ochthebius (s.str.) peisonis Ganglbauer, 1901}

Recorded from eastern Kazakhstan for the first time by Zinchenko (2015).

Kazakhstan: $45^{\circ} 54^{\prime} 04.9^{\prime \prime} \mathrm{N}, 62^{\circ} 08^{\prime} 32.1^{\prime \prime E}$, Kyzylorda Oblast', 15 km NNE Kazalinsk, 70 $\mathrm{m}$, pools near highway, 14 Jun 2015, leg. S. Litovkin, 18 ex. (SLC); 4337'26"N, 68³1'56"E, $\sim 600$ m, Karatau Ridge, 8-10 km N Kentau, Bayaldyr River valley, 2-5 May 2013, leg. S. Litovkin, 2 ex. (1 ex. - SLC, 1 ex. - NMW); 4329'43"N, 70³9'37"E, 375 m, Zhambyl Oblast', vicinity of Ashchykol' (salt lake), brackish pools, 03.06.2015, leg. S. Litovkin, 1 ex. (SLC).

\section{Ochthebius (s.str.) pusillus Stephens, 1835}

Russia (CT): Samara Oblast', Kinel' District, $10 \mathrm{~km}$ SE Malaya Malyshevka, bank of Samara River, 15 Aug 2005, leg. S. Litovkin, 4 ex. (SLC); 53¹3'18"N, 50³3'13"E, Samara Oblast', Volzhskiy District, 1.0-3.5 km SE Alekseevka, waterbodies in Samara River floodplane, different dates, leg. S Litovkin, several dozens of specimens (SLC); $52^{\circ} 55^{\prime} 58.8^{\prime \prime} \mathrm{N}$, 49 38'55.5"E, Samara Oblast', Bezenchuk District, near Chapaevsk, small lake in Chapaevka River flood-plane, 2-4 Jul 2013 and 10 Aug 2015, leg. S. Litovkin, several dozens of specimens (SLC); 5316'39.4"N, 5056'51.6"E, Samara Oblast', Kinel' District, lake near Vertyaevka, 19 Jul 2013, leg. S. Litovkin, 13 ex. (SLC); Ural: Chelyabinsk Oblast', left bank of Ural River, $5 \mathrm{~km} \mathrm{~S}$ Cheka Hill, 15-16 Jul 2007, leg. P. Petrov, 1 ex. (NMW); (ST): $50^{\circ} 57^{\prime} 25.0^{\prime \prime} \mathrm{N}, 54^{\circ} 15^{\prime} 50.3^{\prime \prime} \mathrm{E}, 85 \mathrm{~m}$, Orenburg Oblast', Sol'-Iletsk District, $4.8 \mathrm{~km}$ SE Novoiletsk, oxbow in Ilek River flood-plane, 24 May 2012, leg. S. Litovkin, 4 ex. (SLC); 50 55'45.7"N, 5552'57.7"E, Orenburg Oblast', Akbulak District, 18.5 km SE Akbulak, Karabutak River, 30 Apr 2015, leg. S. Litovkin, 1 ex. (SLC). 


\section{Ochthebius (s.str.) yoshitomii Jäch et Delgado, 2014}

(Figs 9-11)

The correct type locality of this species is: $44^{\circ} 18^{\prime} 50^{\prime \prime} \mathrm{N}, 145^{\circ} 58^{\prime} 56^{\prime \prime} \mathrm{E}$, sea shore, about $3.5 \mathrm{~km}$ SW of the mouth of Severjanka River, Kunashir Island, Kuril Islands, Russian Far East. In the original description, Krugly Cape, $44^{\circ} 00.28^{\prime} \mathrm{N}, 145^{\circ} 39.38^{\prime} \mathrm{E}$, was erroneously listed as type locality.

Russia (FE): $44^{\circ} 18^{\prime} 50^{\prime \prime} \mathrm{N}, 145^{\circ} 58^{\prime} 56^{\prime \prime} \mathrm{E}$, Kunashir Island, Ohkhotsk Sea coast, SW of mouth of Severjanka River, 26 Jul 2013, leg. K. Makarov, 42 adults + numerous larvae (MPU); 4356'33"N, $145^{\circ} 35^{\prime} 20^{\prime \prime} \mathrm{E}$, Kunashir Island, Danilovo, west coast of Lake Peschanoe, 1 Aug 2011, leg. K.

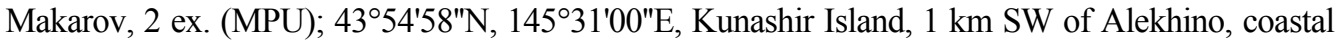

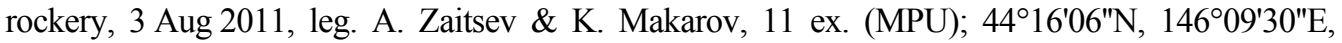
Kunashir Island, mouth of Tiatina River, 6 Sep 2009, leg. A. Prosvirov, 1 ex. (MPU).

Most of the specimens collected by K. Makarov and A. Zaitzev on Kunashir Island were found in filamentous algae in small coastal rock pools with numerous Culicidae larvae, flooded during high tide (Figs 9-11). A smaller number of specimens were collected in marine beach wrack near the mouth of Tyatina River and in lacustrine beach wrack with small pieces of driftwood at Lake Peschanoe.

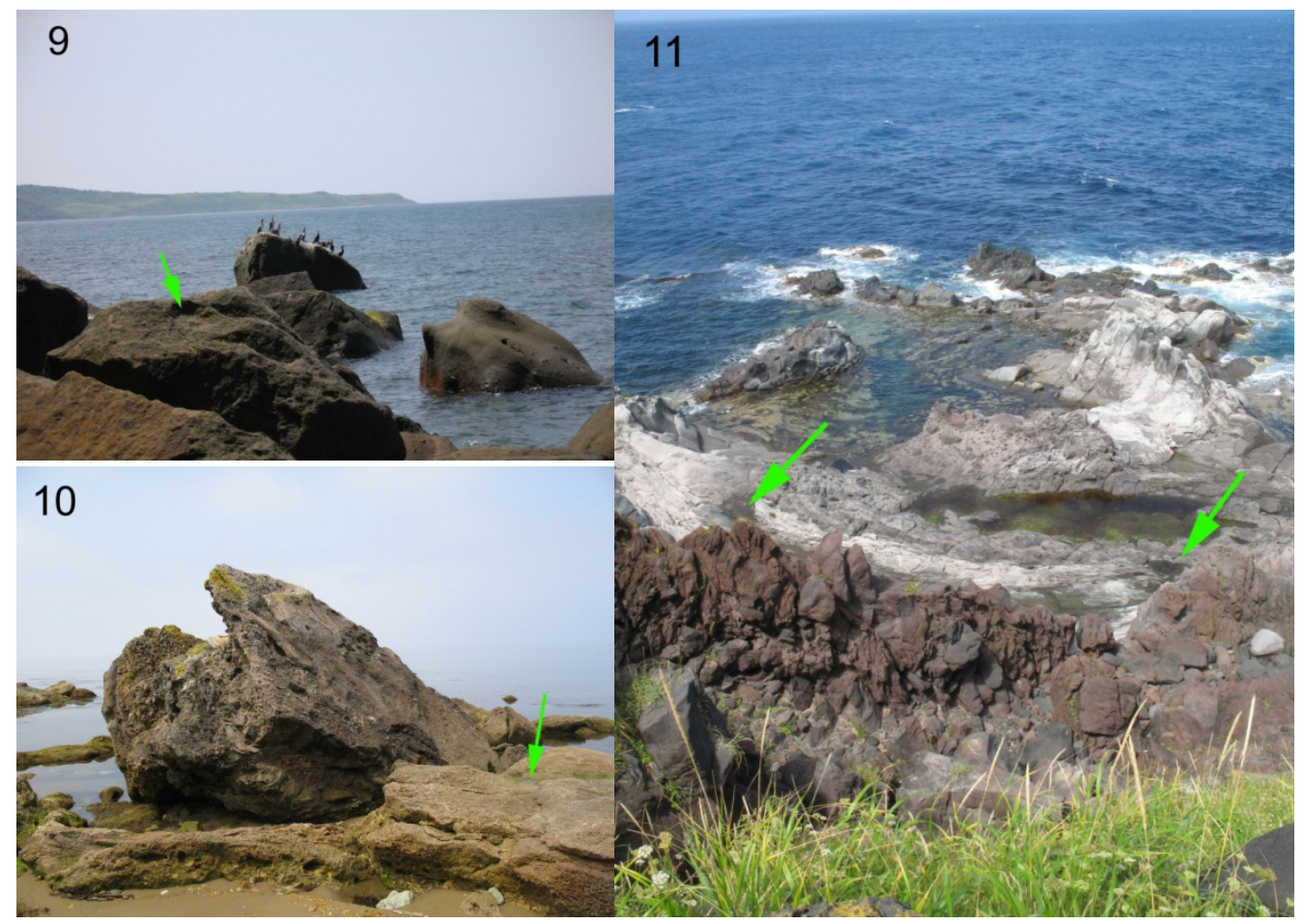

Figs. 9-11. Habitat of Ochthebius yoshitomii Jäch et Delgado, 2014 on Kunashir Island. Photos by K. Makarov. Arrows indicate actual collecting sites; $1 \mathrm{~km} \mathrm{SW}$ Alekhino (9-10), S of mouth of Severjanka River (11).

\section{Ochthebius (s.str.) zugmayeri Kniž, 1909}

Russia (ST): Russia, Astrakhan Oblast', Lake Baskunchak, 5 Jul 2012, leg. D.M. Astakhov, 7 ex. (APC), 25-26 Sep 2014, leg. E.Yu. Mosolova, 2 ex. (APC).

The record for ST in Jäch, Skale (2015) is based on specimens from this location. 
Family Elmidae Curtis, 1830

\section{Stenelmis koreana Satô, 1978}

(Fig. 12)

Russia (FE): Khabarovsk Kray, Bashurovo, Amur River, on UV lamp, 31 Jul2 Aug 1980, leg. V. Belov, 22 ex. (ZMUM).

New for Russia!

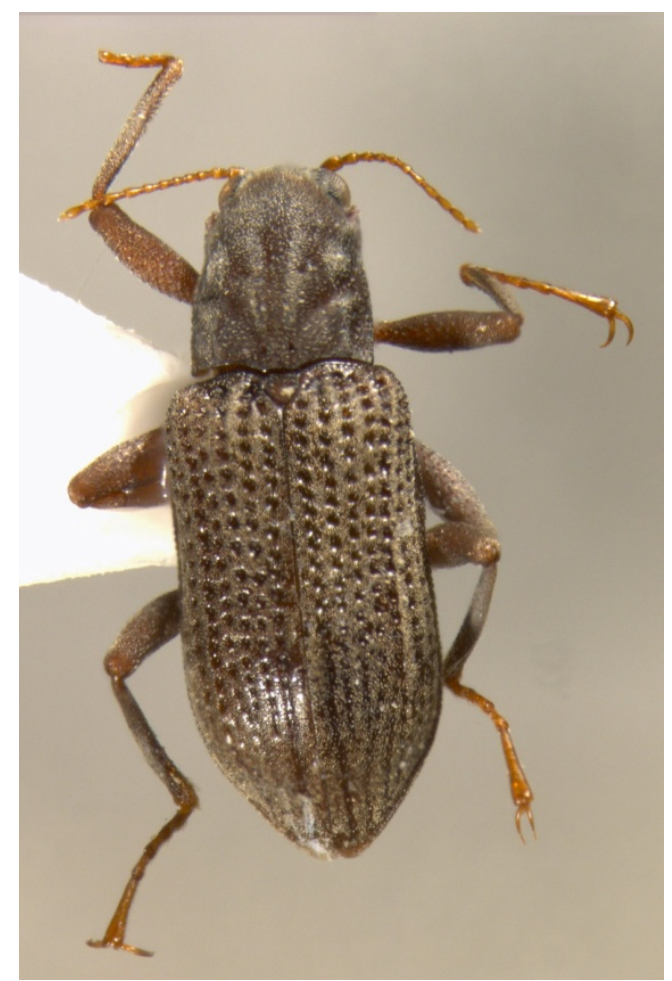

Fig. 12. General habitus of Stenelmis koreana Satô, 1978 from Khabarovsk Kray, ZMUM. Photo by A. Prokin.

\section{Zaitzeviaria brevis (Nomura, 1958)}

Russia (FE): $44^{\circ} 0^{\prime} 31.59^{\prime \prime} \mathrm{N}, 145^{\circ} 40^{\prime} 47.13 " \mathrm{E}$, Kunashir Island, creek Stolbovskoy near hot springs "Stolbovskie" S Cape Stolbchaty, 3 Aug 2013, leg. D. Palatov, 20 ex. (10 ex. - DPC, 8 ex. - APC, 2 ex. - NMW).

This species was recorded from Kunashir by Palatov (2014) under the name Z. gotoi (Nomura, 1959).

\section{Zaitzevia tsushimana Nomura, 1963}

Russia (FE): Primorsky Kray: Ussuriyskyi Reserve and environs of Novochuguievka (Jung et al. 2014).

This species was recorded by Gusakov (2009) from Lazovsky Nature Reserve (Primorsky Kray) under the name Z. rivalis Nomura, 1963. 


\section{DISCUSSION}

For five species the records provided here significantly expand their known ranges. For Ochthebius foveolatus and O. jermakovi the most eastern, for Stenelmis koreana the most northern, for $O$. erzerumi the most northwestern and for $O$. joost $i$ the most northeastern known localities are recorded.

By now, the fauna of Russia includes 66 species of Hydraenidae and 25 species of Elmidae; Ukraine: 40 Hydraenidae, 15 Elmidae; Kazakhstan: 25 Hydraenidae, 4 Elmidae; Uzbekistan: 13 Hydraenidae, 3 Elmidae.

However, the knowledge of the faunal composition of Hydraenidae and Elmidae in Russia and adjacent countries is still quite incomplete. During our studies we detected several undescribed species from Kazakhstan, Russian Caucasus and Abkhazia, which will be described soon.

\section{ACKNOWLEDGEMENTS}

We are grateful to N.B. Nikitsky for the chance to study material of Stenelmis koreana stored in the ZMUM, and all collectors (D. Palatov, Ya. Kovalenko, A. Kovalev, K.V. Makarov, D. Smirnova, A. Silina, I. Fedorov, A. Prosvirov, P. Petrov, D. Astakhov, E.Yu. Mosolova, A. Zaitsev) for providing material for study. We are obliged to K. Makarov for providing ecological information and photographs of the habitat of Ochthebius yoshitomii, and for the photograph of Micragasma paradoxum. Thanks are due to A.M. Shapovalov (St. Petersburg, Russia), D.F. Shovkoon (Buzuluk, Russia), S.V. Kornev (Orenburg, Russia), E.P. Kazakov (Orenburg, Russia) and A. Kutuzov (Borok, Russia) for their help during field work. This study was supported by the Russian Science Foundation, project no. 15-14-10020.

\section{REFERENCES}

GuSAKOV A.A. 2009. Family Dryopidae, subfamily Elminae // Insects of Lazovsky Nature Reserve. Vladivostok: Dalnauka, 138 pp. [in Russian]

JÄCH M. A. 1990. Revision of the Palearctic species of the genus Ochthebius Leach V. The subgenus Asiobates (Coleoptera: Hydraenidae). Koleopterologische Rundschau 60: 37-105.

JÄCH M. A. 1991. Revision of the Palearctic species of the genus Ochthebius VII. The foveolatus group (Coleoptera: Hydraenidae). Koleopterologische Rundschau 61: 61-94.

JÄCH M. A. 1992a. Revision of the Palearctic species of the genus Ochthebius Leach VI. the marinus group (Hydraenidae, Coleoptera). Entomologica Basiliensia 14 (1991): 101-145.

JÄCH M. A. 1992b. Revision of the Palearctic species of the genus Ochthebius Leach X. the punctatus species group (Hydraenidae: Coleoptera). Bulletin et Annales de la Société Royale Belge d'Entomologie 128: 167-195.

JÄCH M. A. 1995. Taxonomic synopsis of the genus Laeliaena Sahlberg, 1900 (Coleoptera: Hydraenidae). Elytron 8: $35-41$.

JÄCH M. A. 2004. Family Hydraenidae Mulsant, 1844. In: LöBL I. \& SMETANA A. (eds), Catalogue of Palaearctic Coleoptera. Vol. 2, pp. 102-122. Apollo Books, Stenstrup, 942 pp.

JÄCH M. A. \& PROKIN A. A. 2005. Faunistic notes on the Hydraenidae, Elmidae, and Dryopidae of the Middle Russian Forest-Steppe Zone (Coleoptera) // Entomological Problems 35 (1): 5-10.

JäCH M. A. \& SKALE A. 2015. Family Hydraenidae Mulsant, 1844. In: LöBL I. \& LÖBL D. (eds.): Catalogue of Palaearctic Coleoptera. Vol. 2/1. Revised and Updated Edition. Leiden-Boston: Brill. P. 130-162.

JunG S. W., JÄCH M. A. \& BAE Y. J. 2014. Review of Korean Elmidae (Coleoptera: Dryopoidea) with description of three new species. Aquatic Insects 36 (2): 93-124.

Litovkin S. V. 2012a. A preliminary review of the fauna of water beetles (Insecta, Coleoptera) of the «Buzulukskyi Bor» National Park. Trudy Orenburskogo REO [Proceedings of Orenburg branch of Russian Entomological Society] 2: 47-53. Russkoe Éntomologicheskoe Obshchestvo, Orenburg. [In Russian with English abstract]

LiTOVKIN S. V. 2012b. Interesting findings of water beetles (Coleoptera: Dytiscidae, Hydraenidae) in the extreme South of the Orenburg region. Trudy Orenburskogo REO [Proceedings of Orenburg branch of Russian Entomological Society] 2: 54-55. Russkoe Éntomologicheskoe Obshchestvo, Orenburg. [In Russian with English abstract] 
Makarov K. V., Matalin A. V. \& Komarov E. V. 2009. Fauna of beetles (Coleoptera) of Elton Lake vicinities. In: A. A. TishKov (ed.), Animals of clayey semidesert in Transvolga region (fauna conspecta and ecological characteristics), pp. 95-134. KMK Scientific Press, Moscow, 164 pp. [In Russian]

Palatov D. M. 2014. New data on the benthic macroinvertebrate fauna in fresh waters of Kunashir island. Vladimir Ya. Levanidov's Biennial Memorial Meetings 6: 509-522. [In Russian with English abstract]

Prokin A. A., Petrov P. N. \& Zhgareva N. N. 2013. Fauna of water beetles (Coleoptera) of the environs of Borok (Yaroslavl Oblast, Russia). In: Hydroentomology in Russia and adjacent countries: Materials of the Fifth AllRussia Symposium on Amphibiotic and Aquatic Insects, pp. 140-144. Filigran, Yaroslavl. [In Russian with English abstract]

SilinA A.Ye. \& PROKIN A. A. 2011. Water Insects (Insecta: Heteroptera, Coleoptera) of Rovensky Natural Park . In: Investigation and protection of natural landscapes: Materials of conference, Volgograd, September 12-15, 2011, pp. 65-73. Planeta, Moscow. [In Russian]

TEMRESHEV I. I. 2012. Materials on the fauna of water beetles (Insecta, Coleoptera) of the State National Natural Park "Altyn-Emel". Vestnik of Kazakh National University. Ecology. No.1 (33): 281-284. [In Russian]

ZINCHENKO V.K. 2015. New records of Hydraenidae beetle family (Hydraenidae) from the Asian part of Russia and Kazakhstan. Euroasian Entomological Journal 14 (3): 201-204. [In Russian with English abstract]

\section{STRESZCZENIE}

\section{[Nowe dane o Hydraenidae i Elmidae (Coleoptera) z Rosji i krajów sąsiednich]}

$\mathrm{W}$ artykule podane są nowe dane z Rosji, Ukrainy, Kazachstanu i Uzbekistanu o rozmieszczeniu 27 gatunków Hydraenidae oraz trzech gatunków Elmidae (Coleoptera). Ochthebius foveolatus Germar, 1824 i Stenelmis koreana Satô, 1978 zostały wykazane z Rosji po raz pierwszy. Inne nowe stwierdzenia to: Ochthebius bernhardi Jäch et Delgado, 2008 (Ukraina), O. depressus Sahlberg, 1900 (Ural), O. erzerumi Kuwert, 1887 (europejska część Rosji i Ural), O. flavipes Dalla Torre, 1877 (Ural), O. jermakovi Orchymont, 1933 (Kazachstan), and O. joosti Jäch, 1992 (Kazachstan i Uzbekistan). Odnotowano także nowe stwierdzenie w Uzbekistanie rzadko rejestrowanego gatunku Laeliaena sparsa Sahlberg, 1900. Praca podaje też dane o nowym stwierdzeniu Ochthebius yoshitomii Jäch et Delgado, 2014 oraz skorygowane dane dotyczące lokalizacji okazów typowych, zawiera również szczegółowe informacje na temat środowiska oraz fotografie jego siedliska na Wyspie Kunaszyr (Wyspy Kurylskie).

Dla pięciu gatunków stwierdzono nowe lokalizacje, które istotnie rozszerzają ich zasięgi, i tak: odnotowano nowe granice wschodnie zasiegów Ochthebius foveolatus i O. jermakovi, granicę północną dla Stenelmis koreana, północno-zachodnią dla O. erzerumi oraz północnowschodnią dla $O$. joosti.

Obecnie fauna Rosji zawiera 66 gatunków Hydraenidae i 25 gatunków Elmidae, Ukrainy odpowiednio 40 i 15, Kazachstanu - 25 i 4 a Uzbekistanu - 13 i 3. 